\title{
For transparency and accountability more is better
}

\author{
To the Editor - The National Institutes \\ of Health (NIH) issued new rules to \\ strengthen the scientific enterprise by \\ promoting transparency and accountability ${ }^{1}$. \\ The agency seeks to ensure that scientific \\ experiments on humans are registered as \\ they begin and reported when they end. \\ These rules are in response to a growing \\ unease about the scientific enterprise, \\ amid numerous reports about lack of \\ transparency, reproducibility and reporting. \\ Recently, three separate studies (including \\ one from NIH investigators) found that
} $\mathrm{NIH}$-funded trials as well as studies led by investigators at prominent academic medical centres in the United States were neither commonly reported nor published within two years of completion and, for many, results were not available up to five years after completion ${ }^{2-4}$.

While the NIH should be lauded for tackling these issues, some scientists have questioned the agency's definition of clinical trials and whether the policy's focus should be narrowed, raising concerns that the new rules will impede research ${ }^{5}$. A principal objection is that their human experiments proceed quickly and require frequent iteration in design, making pre-specification and public registration, in their view, burdensome. However, conducting science in this manner may make it even more important to publicly register the original design of the study and planned statistical methods, including prespecification of outcomes and how the study was changed as data accumulated, along with reporting findings as each iterative version of the study concludes. It is unclear how documentation of human experiments and the reporting of results, tasks that must be part of any laboratory's standard operating procedure, might compromise the ability of scientists to conduct studies.

The concerns of the scientific community are to be respected and we expect the $\mathrm{NIH}$ will work to clarify its intent, its new definition of clinical trials, and implications for training grants and institutional review board approval. However, the goal of these rules is to respect the efforts of humans volunteering to participate in experiments by ensuring that study design, conduct and results can be verified and/or reproduced by others in the scientific community, regardless of the risk posed by the study, whether the science is 'discovery oriented' as opposed to hypothesis testing, or whether the study is intended for pilot or feasibility purposes.

The challenge lies in finding ways to incorporate best practice into workflows and to avoid duplicative work by ensuring that the best practices used for scientific documentation involve public preregistration and results reporting, ensuring that the scientific community is learning from one another. It may be that results of iterative, related experiments could be registered and posted together publicly rather than piecemeal, in the story-based format used by the field for publications. And it may be that formal peer-reviewed publication is not always merited, but that results reporting and/or preprinting is sufficient.

Ultimately, receiving NIH funds and conducting experiments with human participants is a privilege - and with that comes responsibilities. However benign the study, it should be registered and results publicly reported. Such an approach aligns well with the last step of the scientific method, which mandates that scientists report what they find. And this transparency and accountability honours the individuals who have consented to participate in these studies and ensures that their participation has generated knowledge that is shared with others.

\author{
Harlan M. Krumholz $z^{1,2,3 \star}$ and \\ Joseph S. Ross $2,3,4$ \\ ${ }^{1}$ Section of Cardiovascular Medicine, Department of \\ Internal Medicine, Yale School of Medicine, New Haven,
}

CT, USA. ${ }^{2}$ Department of Health Policy and Management, Yale School of Public Health, New Haven, CT, USA. ${ }^{3}$ Center for Outcomes Research and Evaluation, Yale New Haven Hospital, New Haven, CT, USA. ${ }^{4}$ Section of General Internal Medicine and National Clinician Scholars Program, Department of Internal Medicine, Yale School of Medicine, New Haven, CT, USA.

*e-mail:harlan.krumholz@yale.edu

Published online: 22 January 2018 https://oi.org/10.1038/s41562-018-0300-0

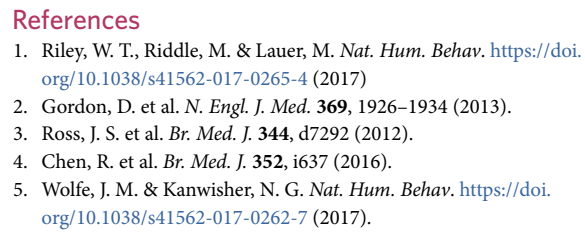

J.S.R. declares funding from the NIH. He has not acted as an advisor for the NIH and was not involved in the planning and implementation of the NIH clinical trials policy. H.M.K has received NIH funding and was previously on the advisory committee to the director of the NIH for several years. H.M.K was not involved in the planning and implementation of the NIH clinical trials policy. In the past 36 months, H.M.K and J.S.R. have had research agreements with Medtronic and Johnson \& Johnson (Janssen), through Yale University, to develop methods of clinical trial data sharing. H.M.K chairs a cardiac scientific advisory board for UnitedHealth; is a participant/participant representative of the IBM Watson Health Life Sciences Board; is a member of the advisory boards of Element Science and Aetna; and is the founder of Hugo, a personal health information platform. J.S.R. has received support through Yale University from Medtronic, Inc. and the Food and Drug Administration (FDA), from the FDA to establish Yale-Mayo Clinic Center for Excellence in Regulatory Science and Innovation (CERSI) programme, from the Blue Cross Blue Shield Association, from the Centers of Medicare and Medicaid Services (CMS) to develop and maintain performance measures that are used for public reporting, from the Agency for Healthcare Research and Quality, from the National Heart, Lung and Blood Institute of the NIH, and from the Laura and John Arnold Foundation to establish the Good Pharma Scorecard at Bioethics International and to establish the Collaboration for Research Integrity and Transparency (CRIT) at Yale. 